\title{
EDUCACIÓN PARA LA DIVERSIDAD EN LA AMAZONIA: ANÁLISIS DE UN PROCESO DE INCLUSIÓN DESDE LA PERSPECTIVA DEL PROFESORADO
}

\section{EDUCAÇÃO PARA A DIVERSIDADE NA AMAZÔNIA: ANÁLISES DE UM PROCESSO DE INCLUSÃO DA PERSPECTIVA DO PROFESSOR}

DOI: http://dx.doi.org/10.5965/1984317815012019119

Ana Luiza Coutinho Leal Universidade Federal do Pará analuizal@ymail.com

\begin{abstract}
RESUMEN
Este estudio busca investigar la inclusión de niños especiales en las clases de cuatro escuelas en Belém. El problema de investigación es: ¿Cómo se da la inclusión del niño especial en las clases de la Educación Infantil y de la Enseñanza Primaria en Belém? La investigación fue desarrollada entre los años de 2010 y 2011 , es una investigación puramente cualitativa y de carácter descriptivo y los datos fueron obtenidos a través de cuestionarios, entrevistas y observación de las clases. El estudio tuvo como objetivo general analizar el proceso de inclusión del niño especial en la enseñanza reglada en Belém, y como objetivos específicos verificar cómo la diversidad ha sido tratada en las propuestas curriculares en cuestión, identificar el nivel de formación de los docentes que trabajan con esos alumnos e investigar las condiciones de organización de las escuelas locales para acoger a esos niños. La investigación evidenció que hay una distancia considerable entre lo que se habla respecto de la inclusión y la realidad y eso es motivado por la falta de capacitación de los docentes, la precariedad de la infraestructura de las escuelas y la ausencia de un currículo flexible que atienda a una población diversificada.
\end{abstract}

Palavras-chave: Educación. Inclusión. Diversidad. Currículo.

\section{RESUMO}

Este estudo busca investigar a inclusão da criança especial nas salas de aulas de quatro escolas de Belém. A pergunta que constitui nosso problema de investigação é: Como se dá a inclusão da criança especial com déficit cognitivo nas salas de aulas regulares da Educação Infantil e da Educação Primária em Belém? A pesquisa foi desenvolvida, nos anos de 2010 e 2011 e é uma investigação qualitativa de caráter descritivo, e os dados foram obtidos através de questionário, entrevista e observação das aulas. O estudo teve como objetivo geral analisar o processo de inclusão da criança especial dentro do ensino regular em Belém e como objetivos específicos verificar como a diversidade tem sido contemplada nas propostas curriculares em questão, identificar o nível de formação profissional dos docentes que trabalham com esses alunos e investigar a infraestrutura das escolas locais para acolher essas crianças. A pesquisa evidenciou que há uma grande diferença entre o que se fala a respeito da inclusão e a realidade e isso é motivado pela falta de capacitação dos docentes, a precariedade da infraestrutura das escolas para lidar com a diversidade e a ausência de um currículo flexível que atenda a uma população diversificada.

Palavras-chave: Educação. Inclusão. Diversidade. Currículo. 


\title{
EDUCATION FOR DIVERSITY IN THE AMAZON: ANALYSIS OF AN INCLUSION PROCESS FROM THE TEACHER'S PERSPECTIVE
}

\begin{abstract}
This study aims to investigate the inclusion of children with special educational in regular classrooms in Belém. The question that constitutes our research problem is: How does the inclusion of children with special educational needs caused by cognitive defects is possible in regular classroom of Elementary Education in Belém? The research was developed in the years of 2010 and 2011, is a descriptive qualitative research and the information has been obtained through a questionnaire, interviews and class's observation. The study aimed to analyze the inclusion of special children in formal education in Belém. The specific objectives of our investigation consisted in checking how the diversity has been measured in the curricular proposals, identify the level of aptitude of teachers working with these students and investigate the conditions of organization and infrastructure of local schools to accommodate these children. The research illustrated that there is a big distance among what people say about the inclusion and the reality and this is motivated by the lack of training for teachers, the dreadful local infrastructure schools to work with diversity and the absence of a flexible curriculum that serves a diversified population.
\end{abstract}

Keywords: Education. Inclusion. Diversity. Curriculum.

\section{INTRODUCCIÓN}

La educación para la diversidad implica cambios y transformaciones en el nivel de las estrategias, contenidos, abordajes y estructuras, basadas en una visión común que alcance a todos los niños de la misma faja etaria y en la convicción de que es de responsabilidad del sistema regular de enseñanza educar a todos los alumnos.

En este estudio abordamos la inclusión de los alumnos con necesidades educativas especiales derivadas del déficit cognitivo en algunas escuelas de la red pública y privada en Belém, ciudad que integra la Región Amazónica, ubicada en el norte de Brasil, en el Estado de Pará.

Siendo así, para investigar ese tema, de acuerdo con los marcos teóricos, tenemos como objetivos analizar el proceso de inclusión del niño con necesidades educativas especiales derivadas del déficit cognitivo en los salones de clase de la Educación Infantil y de la Enseñanza Primaria en Belém, además de verificar cómo la diversidad ha sido contemplada en las propuestas curriculares en cuestión, bien como identificar el nivel de formación profesional de los docentes que trabajan con alumnos 
con necesidades educativas especiales e investigar las condiciones de organización y de infraestructura de las escuelas locales para acoger a esos niños.

En esa investigación utilizamos el modelo no-experimental, transversal descriptivo y cualitativo y la estrategia de pesquisa es el estudio de caso o, más específicamente, el estudio de multicasos, puesto que se trata de cuatro casos donde cada uno se caracteriza por sus propias particularidades.

Los instrumentos de colecta de datos fueron el cuestionario mixto con los profesores de los niños especiales observados, la entrevista estructurada con las directoras y técnicas especializadas de las escuelas investigadas y la observación sistematizada de los niños especiales.

Dentro del marco referencial, dividimos la investigación en los siguientes tópicos: Educación y diversidad: La diversidad en el ámbito educativo; La educación inclusiva x la educación especial; Nuevas perspectivas: la educación concebida desde la perspectiva de la Escuela Inclusiva y La formación del profesorado para una escuela inclusiva.

Dentro del marco metodológico definimos el diseño metodológico de ese estudio que comprende los siguientes tópicos: La investigación de campo; Diseño metodológico; Delineación del estudio (problema y objetivos; población y muestra profesores, alumnos y escuelas); Instrumentos de colecta de datos.

En el último tópico, presentamos y analizamos los resultados de la investigación de campo y, finalmente, concluimos la misma relacionando los resultados con los objetivos de la investigación y presentando recomendaciones de acciones para que el proceso educacional inclusivo tenga éxito en Brasil.

\section{EDUCACIÓN Y DIVERSIDAD}

Educar para la diversidad no es posible sin una real concientización de que la diversidad es el elemento que acerca a las personas en cuanto individuos.

Una educación que pretenda atender a la diversidad debe ser enfocada desde la perspectiva de la igualdad de oportunidades como preludio para la participación efectiva de los individuos en las diversas actividades de la sociedad, considerando la 
justicia y la equidad como elementos sin los cuales la inclusión no dejará de ser una utopía que pasa de generación en generación.

\subsection{La diversidad en el ámbito educativo}

Para iniciar este trabajo es esencial conocer algunos conceptos que lo fundamentan, como los de educación, inclusión, diversidad y educación especial.

Educación es una forma de reproducción de los saberes que componen una cultura, por tanto, la educación de una sociedad tiene identidad propia y sus agentes reproducen ideologías que reflejen la sociedad como un todo.

Inclusión es un proceso que consiste en ingresar alumnos con necesidades educativas especiales en los salones regulares de enseñanza, considerando las necesidades de todos los alumnos y produciendo un cambio en la perspectiva educacional suprimiendo la división de los sistemas escolares en modalidades de enseñanza especial y de enseñanza reglada.

Según Mantoan (2008), inclusión es estar con; es interactuar con el otro, o sea, incluir implica tomar en cuenta a los distintos grupos que conforman las sociedades y considerar a cada individuo que los conforma parte de un todo diverso. Incluir significa entender que las culturas son diferentes y que, por lo tanto, las personas también lo son, pero que no por eso pertenecientes a mundos diferentes.

En el término diversidad confluyen la variedad y convivencia de ideas, características o elementos diferentes entre sí de determinado asunto, situación o ambiente. La idea de diversidad está unida al reconocimiento, al respeto y a la valoración de las diferencias como factor para la coexistencia armoniosa de las varias formas posibles de no semejanzas.

Educación especial es:

Toda educación debe ser especial en el sentido de atender a las características y necesidades especiales de cada alumno. En esa línea, la educación especial sería un grado o una forma a más de individualización de la enseñanza, de adaptación de los programas, de adecuación de los métodos y de los recursos en cada caso concreto (SÁNCHEZ, 1988 apud TORRES GONZÁLEZ, 2002, p. 67). 
Se observa que la concepción más actual aborda las demandas educativas de los alumnos en general y no sólo de los "deficientes" que necesitan de una educación especial, o sea, aborda las necesidades específicas de todo alumno que, a lo largo del proceso educativo, requiere una atención mayor que sus colegas de la misma edad por presentar problemas de aprendizaje, sean de forma temporaria o permanente.

\subsection{La educación inclusiva x la educación especial}

La educación inclusiva no debe ser confundida con la educación especial, la cual se presenta en una gran variedad de formas incluyendo escuelas especiales, unidades pequeñas y la integración de los niños con apoyo especializado.

La enseñanza especial, como ya vimos, es un sistema particular de educación de alumnos especiales fuera de la enseñanza regular, basado en la creencia de que las necesidades de esos alumnos no pueden ser suplidas por las escuelas regulares.

La educación inclusiva, al contrario, considera la diversidad inherente a la especie humana, buscando reconocer y atender las necesidades educativas especiales de todos los alumnos, en las aulas comunes, en un sistema regular de enseñanza, de modo de promover el aprendizaje y el desarrollo personal de todos.

Para Sánchez (2005, p. 8), la educación inclusiva fue vista, primeramente "como una innovación de la educación especial, pero, progresivamente, fue expandiéndose en todo el contexto educativo como un intento de que una educación de calidad alcanzara a todos".

Siendo así, el derecho a la diferencia en las escuelas deconstruye el sistema escolar actual que es excluyente, dado que posee mecanismos de producción de la identidad y de la diferencia.

La enseñanza inclusiva es un proceso social donde todos los educandos con discapacidad o con disturbios de aprendizaje tienen el derecho a la escolaridad lo más cerca posible de "lo normal". El objetivo a ser alcanzado es la integración de ese alumno en la comunidad.

Esa enseñanza es regida por el principio de que todos deben aprender juntos, siempre que sea posible, considerando las diferencias individuales entre alumnos de la misma edad en aulas heterogéneas. 
Pero para que haya esa inclusión, la red regular de enseñanza, tanto pública como privada, debe proporcionar programas educacionales apropiados a las necesidades y capacidades del alumno; asimismo ofrecer asistencia y apoyo de que necesitan los alumnos especiales.

En ese sentido,

incluir alumnos con deficiencias importantes en los grupos de educación regular eleva la consciencia de cada aspecto interrelacionado de la escuela como una comunidad: sus límites, los beneficios a sus miembros, sus relaciones internas, sus relaciones con el ambiente externo y su historia (STAINBACK; STAINBACK, 1999, p. 48).

Por lo tanto, el gran mérito de la escuela inclusiva es proporcionar educación de calidad a todos, así como eliminar actitudes discriminatorias, creando comunidades escolares que abriguen a todos y, de esa forma, hacer que la sociedad sea más igualitaria.

\subsection{Nuevas perspectivas: la educación concebida desde la perspectiva de la Escuela Inclusiva}

La educación especial ha sido considerada durante mucho tiempo una modalidad educacional cuyo objetivo era trabajar las discapacidades de los individuos, y su objeto de estudio era, como se puede deducir, personas que presentaban alguna discapacidad que les impedía de adaptarse a las propuestas curriculares regulares. Esa forma de concebirla está siendo repensada, puesto que en los últimos años la sociedad se ha ido concientizando poco a poco de que el concepto de educación especial es mucho más amplio de lo que antaño se pensaba.

Los cambios que se vienen observando en la educación y, por consiguiente, en la educación especial, nos hacen ver, siguiendo el razonamiento de Torres González (2002, p. 91), que "estamos pasando de las estructuras a los procesos; de la segregación a la flexibilidad y a la adaptación del sistema educacional", aunque, si queremos ser justos a la hora de aproximarnos a la educación especial, sigue el autor, "debemos señalar que ambos enfoques coexisten actualmente".

En cualquier caso, la educación especial es concebida hoy como un subsistema de la educación general y que camina de la mano con ésta. 
Su objetivo es, en líneas generales, potenciar y asegurar la consecución de los principios que defienden la equiparación de oportunidades de los individuos que necesitan de atención especial para su buen desarrollo educacional en todos los niveles de formación. De ahí la idea de que la educación va más allá de ser un mero elemento de relación entre enseñanza y aprendizaje; la educación es, de esa perspectiva, un conjunto de herramientas que los individuos disponen para mejorar su desarrollo integral. De esa forma, el desarrollo de la persona puede ser considerado como fin último de la educación.

En este momento la educación es considerada un derecho básico humano, como establecido en la Declaración Universal de los Derechos Humanos de 1948. Trascender el campo de la mera formalidad y colocar en práctica los principios definidos en los grandes foros que discuten la cuestión de la educación como derecho es el desafío para el fututo, especialmente cuando se trata de la educación especial.

Entre los derechos elementales que tienen relación con la educación, podemos destacar el derecho a la igualdad de oportunidades y el derecho a la participación y a la no discriminación. Pese a que cada uno de esos derechos constituye un proceso diferente y difícil de llevar a cabo hoy en día, eso no debe paralizarnos ante las necesidades constantes de cambios en el ámbito de la educación especial, cuya finalidad debe ser hacer que la escuela se convierta en un lugar centrado en el sujeto, donde los individuos sean realmente sujetos de su propio proceso educacional, y que la educación sea el instrumento potenciador de su desarrollo como personas.

Para que el derecho a la educación se haga efectivo es necesario, entre otras acciones, hacer que la propuesta de un currículo abierto y flexible se concrete, puesto que el diseño del currículo es esencial para que se lleve a cabo acciones en el sentido de incluir a todos en los programas de educación y, de esa forma, lograr el equilibrio entre lo que consideramos común y lo que consideramos diversificado, y, de ese modo, encontrar las condiciones de igualdad, que es uno de los principios que rigen la idea de educación inclusiva. Esa idea de flexibilidad curricular contrarresta la antigua concepción según la cual los individuos eran los que tenían que adaptarse a los programas educacionales ofrecidos, que en líneas generales eran homogeneizadores. 


\subsection{La formación del profesorado para una escuela inclusiva}

A lo largo de esta investigación, hemos observado que las exigencias de la respuesta educativa a la diversidad implica la necesidad de la formación de profesionales capaces de realizar determinadas actividades, a saber, planificar situaciones de aprendizaje, realizar adaptaciones curriculares, elaborar pautas de trabajo en equipo, entre otras actividades decisivas para el éxito o fracaso del proceso educativo.

Según Torres González (2002), el profesor debe crear un ambiente propicio para la interacción y la cooperación, incentivar los alumnos para que mejoren su autoestima y el reconocimiento, aceptar las diferencias como normales y afrontar las diferencias entre los docentes a través de actividades en equipo.

En ese contexto, Canen y Xavier (2005) afirma que, en el caso de la educación, la cuestión de la discriminación de los prejuicios que son asociados a ella pasan a exigir respuestas, a fin de preparar las futuras generaciones para trabajar en comunidades académicas cada vez más desiguales.

De ese modo, se puede afirmar que la escuela debe evolucionar junto con la sociedad democrática, y ese desarrollo puede ser llevado a cabo a través del trabajo en equipo del profesorado.

Pero para que ocurra un progreso en la escuela, tenemos que hablar de un antes y de un después en la educación, puesto que en el proceso educativo intervienen otros factores que influyen en el desarrollo personal del niño.

Para buscar una educación de calidad, es necesario "generar la conciencia de que todos los niños tienen los mismos derechos y necesidades, por lo que hacer posible una igualdad de oportunidades, rechazando actitudes negativas" (REYES REBOLLO, 2010, p. 91), puesto que la concepción de una nueva escuela consiste en que esta sea capaz de ofrecer la ayuda pedagógica que el alumno necesite.

Sabemos que la diversidad es un hecho inherente al desarrollo humano y, por ese motivo, la educación debe hacer que todos los alumnos, aun aquellos que presentan una discapacidad, aprendan juntos.

Villa y Thousand (1995 apud SÁNCHEZ, 2005, p. 11) aseveran que los educadores que se arriesgan a educar niños con deficiencias argumentan que esos 
alumnos "son un regalo para la reforma educativa", pues esos alumnos compelen los docentes a romper el paradigma de la educación tradicional y los obliga a buscar nuevas formas de enseñar.

Según Figueiredo (2008, p. 141), la formación de profesores implica una redefinición de sus competencias y de las funciones que les son atribuidas, tomando en cuenta "principios éticos, políticos y filosóficos que les permitan comprender su papel y el de la escuela frente al desafío de formar una nueva generación capaz de responder las demandas de nuestro siglo".

De modo que, para hablar de la formación del profesorado es necesaria una conciencia de que en los cursos de licenciatura de las universidades brasileñas hay que enfatizar la responsabilidad que los alumnos tendrán como futuros docentes del sistema educativo nacional y de su influencia en el proceso de enseñanza aprendizaje, que puede constituir "un elemento fundamental a la hora de moldear la enseñanza" (REYES REBOLLO, 2010, p. 96).

Por todo que fue dicho, podemos afirmar que el sistema educativo es considerado el formador del futuro ciudadano, y para que el profesor no se quede retrasado en ese ámbito, él debe tener una formación adecuada, para que pueda evolucionar, trabajando en equipo con la comunidad educativa y en favor de una escuela inclusiva que ofrezca una educación de calidad para todos.

\section{LA INVESTIGACIÓN DE CAMPO}

La relevancia social de la presente investigación está en discutir la integración de los alumnos especiales y las implicaciones prácticas, como despertar la concientización de la sociedad sobre el modo en que la diversidad ha sido contemplada en las propuestas curriculares en cuestión.

\subsection{Diseño metodológico}




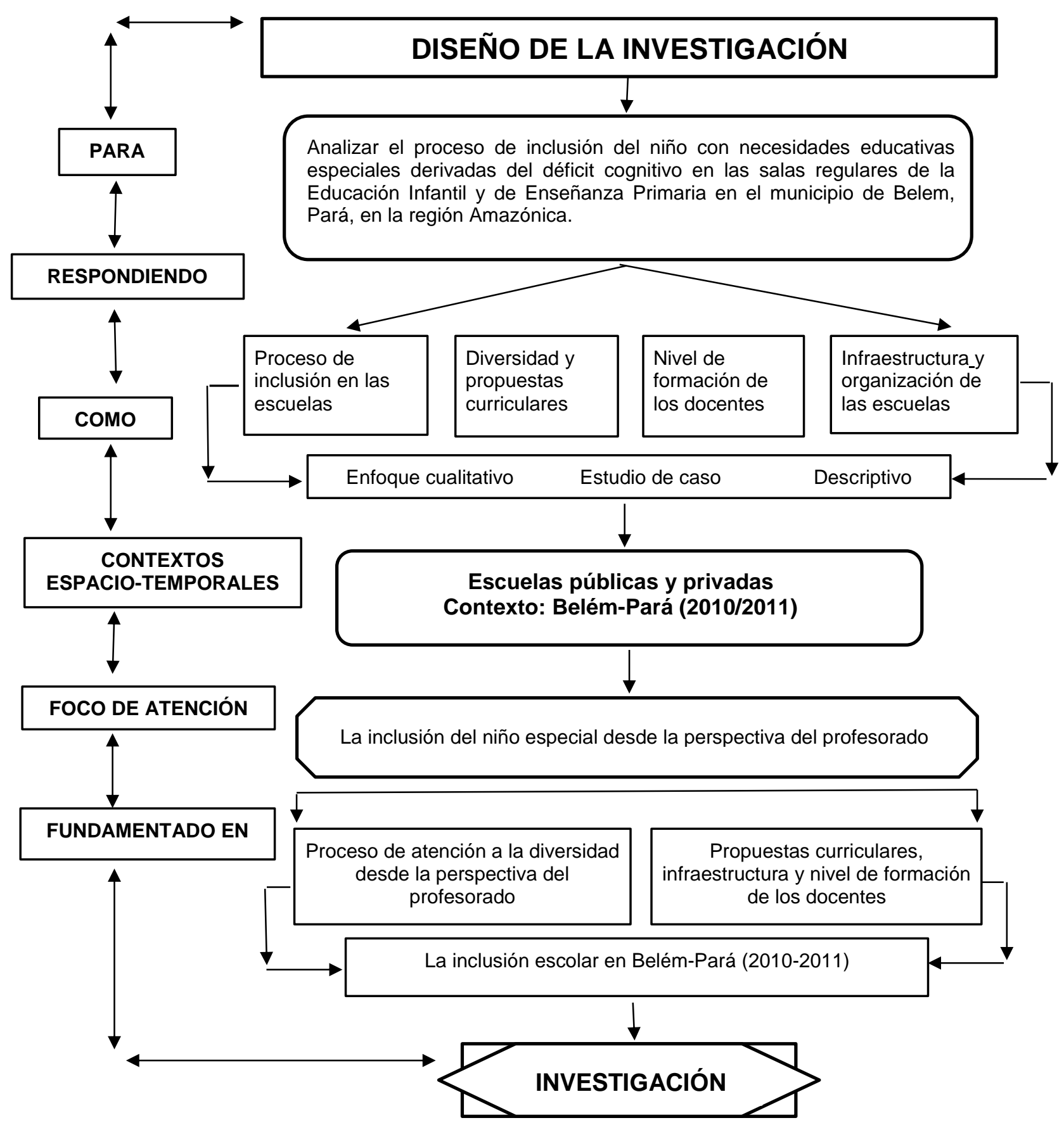

Fuente: Elaboración propia

\subsection{Delineación del estudio}

\subsubsection{Problema y objetivos}


El problema de una investigación tiene que ser detectado a raíz de las lecturas, de las experiencias y del aprendizaje, es decir, de la vivencia intelectual del investigador.

De ese modo, tenemos el siguiente problema de investigación a ser estudiado: ¿Cómo se da la inclusión del niño con necesidades educativas especiales derivadas del déficit cognitivo en las clases regladas de la Educación Infantil y de la Enseñanza Primaria en el municipio de Belém, Estado de Pará, en la Amazonia?

En ese sentido, cuestionamos, además: ¿Cómo los profesores han acogido las diferencias presentes en el cotidiano de las escuelas paraenses? ¿Cómo nuestras escuelas, orientan los profesores para tratar con las diferencias presentes en el salón de clase? ¿Cuáles son las condiciones de organización de las escuelas para trabajar con niños especiales?

De ese modo, tenemos como objetivo general analizar el proceso de inclusión del niño con necesidades educativas especiales derivadas del déficit cognitivo en los salones reglados de la Educación Infantil y de la Enseñanza Primaria en Belém y tenemos como objetivos específicos: verificar cómo la diversidad ha sido contemplada en las propuestas curriculares en cuestión; identificar el nivel de formación profesional de los docentes que trabajan con alumnos con necesidades educativas especiales; investigar las condiciones de organización y de infraestructura de las escuelas locales para acoger a esos niños.

\subsubsection{Población y muestra: profesores, alumnos y escuelas}

Esa investigación fue realizada en Brasil, en la Región Amazónica, en el Estado del Pará, en el municipio del Belém, ciudad considerada la "metrópolis de la Amazonia".

Belém posee más de 1.000 escuelas, entre públicas y privadas. Esa pesquisa se realizó en cuatro escuelas de la red reglada de enseñanza, siendo dos públicas estatales y dos privadas.

De las dos escuelas públicas escogidas, una se ubica en un barrio periférico y otra en el centro de la ciudad. 
Entre las dos escuelas privadas, una se ubica en un barrio pobre y la otra en un barrio central de Belém.

La primera escuela, que llamaremos de "Escuela 1" hace parte de la red pública estatal reglada de enseñanza de Belém, capital del Estado y fue pesquisada entre los meses de agosto y diciembre de 2010.

La segunda escuela hace parte de la red privada de enseñanza de la ciudad de Belém y fue pesquisada también en agosto y diciembre de 2010 y la llamaremos de "Escuela 2".

La tercera escuela pertenece a la red privada, fue pesquisada entre los meses de enero y abril de 2011 y será llamada de "Escuela 3".

Durante el mismo período pesquisamos la "Escuela 4", que pertenece a la red pública estatal.

En la Escuela 1, en la enseñanza primaria, en el turno de la mañana hay tres niños especiales y de los tres sólo nos fue permitido observar a uno de ellos.

En la Escuela 2, en la educación infantil en el turno de la mañana hay cinco niños especiales, tres de ellos con déficit cognitivo, que es el tema de ese trabajo, y los tres fueron observados.

En la Escuela 3, en la educación infantil, en el turno de la tarde, hay tres niños especiales y todos fueron observados.

En la Escuela 4, en el turno de la mañana, en la enseñanza primaria, hay cuatro niños especiales, tres con deficiencia mental y una con deficiencia física, que no es objeto de ese estudio. Siendo así, observamos a dos niños.

El universo de esa investigación es, de cuatro escuelas, quince niños especiales, quince profesores, cuatro directoras y tres personas del sector técnico especializado.

De los quince niños que hacen parte del universo de esa pesquisa, nos fue posible observar a nueve niños, aplicar cuestionarios a doce profesores y entrevistar cuatro directoras y tres técnicos especializados, siendo esa la muestra de este estudio.

\subsubsection{Instrumentos de colecta de datos}


En el presente estudio aplicamos instrumentos de colecta de datos de esencia cualitativa, por ser ese el enfoque en vigor.

Existen diversas técnicas de colecta de datos y, entre ellas, escogemos el cuestionario (para los docentes), la entrevista (para los directores y equipo técnico), y la observación de los niños especiales.

\section{PRESENTACIÓN Y ANÁLISIS DE LOS RESULTADOS DE LA INVESTIGACIÓN DE CAMPO}

Barros (2005, p.86) afirma que "analizar significa buscar el sentido más explicativo de los resultados de la investigación" y está estrechamente ligado a la comprobación y a la renovación de teorías científicas.

En la investigación cualitativa, obtenidos los datos, el investigador tendrá delante de sí respuestas que necesitan ser organizadas, para que puedan ser analizadas e interpretadas.

La interpretación está ligada al análisis y consiste en la capacidad de volverse a síntesis sobre los datos, entendiéndolos en relación a otros estudios ya realizados en la misma área.

Partiendo de esa premisa, presentamos en los tópicos siguientes el resultado de la pesquisa de campo.

\subsection{Educación inclusiva en Brasil}

Por lo que es posible observar, a partir de los datos de la Figura 1, 18\% de la muestra cree que la educación inclusiva es viable; 18\% afirma ser pedagógicamente correcta; $18 \%$ cree ser significativa si el compromiso es serio; $18 \%$ afirma ser inviable, pues los profesores no tienen capacitación (formación adecuada) para trabajar con los niños con necesidades especiales, así como las escuelas necesitan adecuarse físicamente; $28 \%$ afirma representar un avance, aunque falte mucho a ser hecho. 


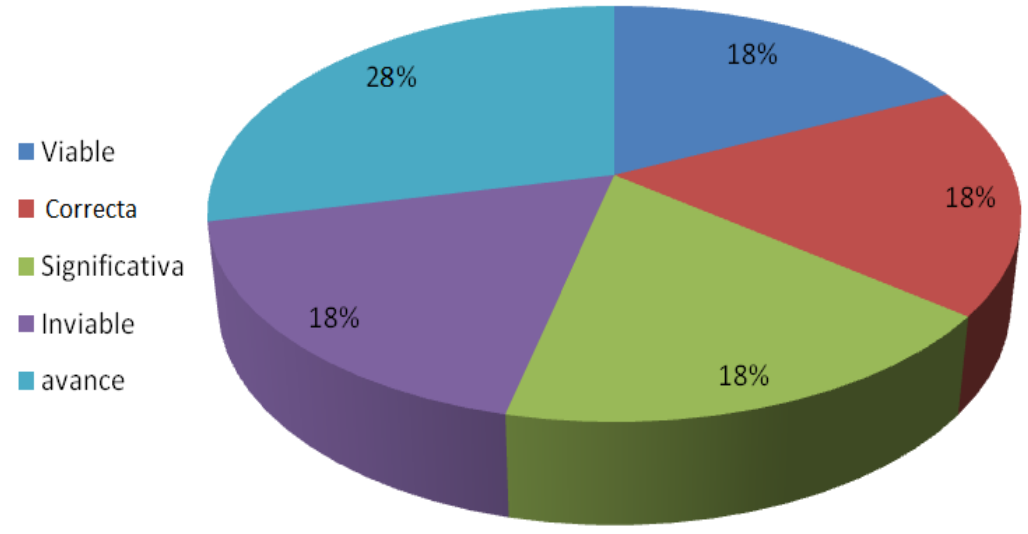

Figura 1 - Opinión sobre la educación inclusiva en Brasil. Fuente: Investigación de campo - Elaboración propia.

Además de las afirmaciones arriba, los profesores afirman que el mismo, de un modo general, aún presenta serias dificultades, tanto de orden teórico, como metodológicos; la escuela, en cuanto institución social pública tiene el deber de ofrecer una enseñanza de calidad para mejor atender las diferencias individuales en la perspectiva de la ciudadanía; la educación se encuentra en el camino de la inclusión, pero mucho aún debe ser hecho, principalmente con relación a la capacitación de los profesores; el proyecto de inclusión social es viable, pero requiere una mayor participación de la sociedad, de los profesores y de las entidades de gobierno.

\subsection{Aspectos positivos de la educación inclusiva}

Con relación a los aspectos positivos en la experiencia con la educación inclusiva, se destaca en la Figura 2 que: $44 \%$ de la muestra apunta la solidaridad con los niños; 33\% apunta el respeto por el niño con deficiencia, inserido en las clases regulares; $6 \%$ apunta la capacitación de los profesores; $6 \%$ apunta la total adaptación del niño con discapacidad en los salones de clases regulares y $11 \%$ destaca, entre los puntos positivos, que el niño con necesidades especiales ha aprendido a comportarse en grupo. 


\section{n Solidaridad}

nespeto

- Capacitación

- Adaptación

- Comportamiento

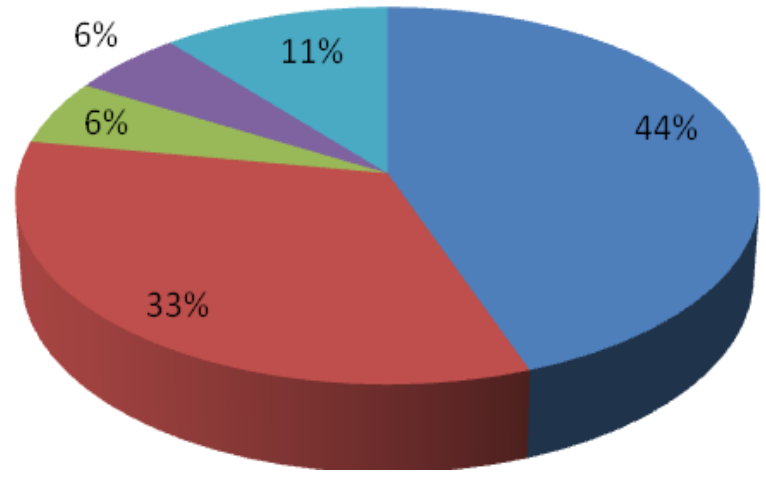

Figura 2 - Aspectos positivos observados en la experiencia de la inclusión. Fuente: Investigación de campo Elaboración propia.

Vale resaltar que además de los ítems arriba, los profesores opinaron de la siguiente forma: las escuelas ya presentan un trabajo accesible de enseñanza para todos; creen que es importante la proximidad con la diferencia, demostrando que todos tienen derecho a espacio y oportunidades; aumenta la autoestima de las personas con necesidades especiales a partir del momento en que notan que son capacitadas para aprender cosas nuevas; quiebra de barreras en la sociedad y la socialización concreta del saber.

Dentro de ese contexto, Mantoan (2008) afirma que, entre los beneficios que la inclusión nos trae, el principal de todos es vivir la experiencia de la diferencia, puesto que ella posibilita que los diferentes ocupen su espacio en la sociedad, y a los alumnos "normales", la oportunidad de valorizar el otro. Además, para los profesores, el beneficio mayor está en garantizar a todos el derecho a la educación.

\subsection{Aspectos negativos relativos a la inclusión}

Dentro de los datos apuntados por la muestra que compone la presente pesquisa, se destacan en la Figura 3: falta de capacitación de los profesores, 46\%; falta de soporte técnico y apoyo especializado por parte de la escuela, 38\%. Además de los ítems citados, otros aspectos fueron también relatados, como por ejemplo: ausencia de infraestructura en las escuelas; necesidad de capacitación continua y apoyo a los profesores. 


\section{Falta de capacitación}

- Falta de soporte técnico

El niño especial retra sala clase

- La escuela no prepara

El niño especial se siente diferente en

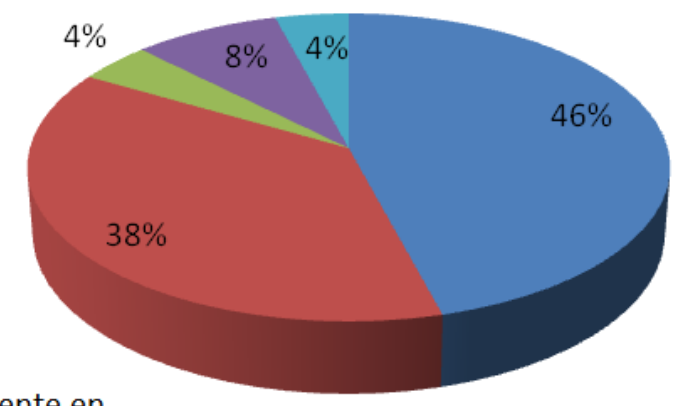

una clase inclusiva

Figura 3 - Aspectos negativos relativos a la inclusión. Fuente: Investigación de campo - Elaboración propia.

Mantoan (2005, p. 27) afirma que "nuestra convivencia con las personas con deficiencia en las escuelas comunes es reciente y genera todavía mucha aprehensión entre los que la componen".

El desafío de la inclusión para los profesionales que actúan a servicio de la mejoría de la calidad de vida humana es proyectar artefactos y lanzar propuestas que no se destinen solamente a un grupo restricto de personas. Siendo así, la inclusión nos lleva a avanzar más, dado que para atender a sus preceptos tenemos que alcanzar situaciones de equilibrio general, las grandes y tan anheladas soluciones que alcancen fines cualitativamente más evolucionados.

\subsection{Evolución del niño con necesidades especiales al ingresar en la escuela regular}

Con relación a la evolución del niño portador de necesidades especiales al ingresar en la escuela, se resaltan las siguientes opiniones (Figura 4): cree que el niño con necesidades especiales que frecuenta la escuela reglada evoluciona rápidamente debido a la interacción con el niño sin necesidades especiales, 46\%; cree que el niño con necesidades especiales no evoluciona por falta de capacitación de los profesores y apoyo técnico por parte de la escuela, 38\%; cree que debería haber una clase especial dentro de la escuela reglada para que el alumno discapacitado pudiera evolucionar, $8 \%$. 
- Evoluciona rápidamente

- No evoluciona

- Debería estudiar en una escuela especializada

- No evoluciona y retrasa la clase

- Debería haber clase especial en la escuela

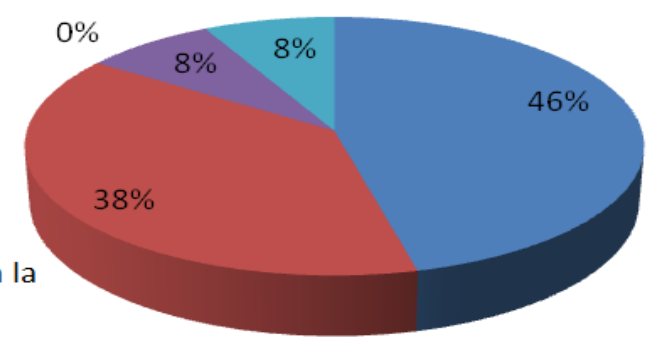

Figura 4 - Observación del niño con necesidades especiales en la escuela. Fuente: Investigación de campo Elaboración propia.

Además de los posicionamientos arriba, se destaca, según los profesores, que la convivencia del niño con necesidades especiales con otros niños favorece el desarrollo del mismo, aunque éste sea a largo plazo, teniendo en cuenta las limitaciones presentadas por el niño.

En ese sentido, Torres González (2001, p. 77) afirma que:

en la actualidad, cuando hablamos de deficiencias, dificultades y la posibilidad de rehabilitarlas, nos adentramos en la teoría de la modificabilidad cognitiva. Los seres humanos pueden modificarse a sí mismos si lo desean, y estos cambios pueden lograrse a niveles no pronosticados,

o sea, el niño especial debe evolucionar interactuando con niños sin discapacidades.

\subsection{Integración e interacción del niño con necesidades especiales}

En lo que se refiere a la forma cómo ocurre el proceso de integración e interacción del niño con necesidades especiales en el salón de clase, se destaca en la Figura 5 que el niño interactúa con los otros con facilidad, 45\%; la integración es difícil debido a problemas de comportamiento del niño, 27\%; el niño en poco se diferencia de los demás niños, por eso se integra bien en el grupo, $28 \%$. 

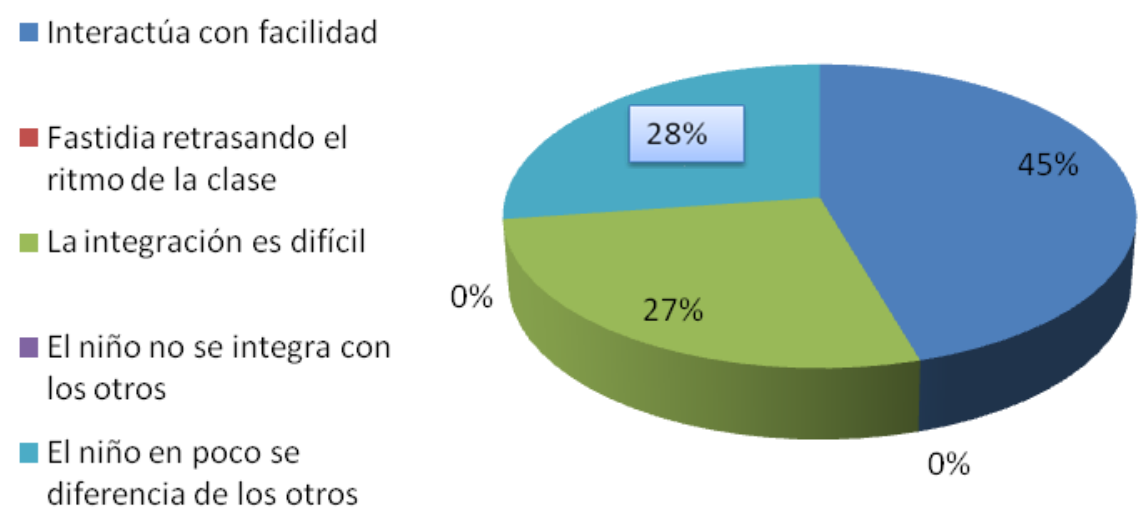

Figura 5 - Proceso de integración e interacción del niño con necesidades especiales en el salón de clase. Fuente: Investigación de campo - Elaboración propia.

Entre otras informaciones obtenidas junto a los profesores, se resalta que el niño especial interactúa con los demás niños en la medida en que es trabajado el grupo de forma que éste valorice el nuevo componente.

La integración de todos en los sistemas normales de educación tiene su fundamento en el hecho de que, para la autora, la educación inclusiva no es esencialmente diferente de la educación común, sino "una visión diferente de la educación común basada en la heterogeneidad y no en la homogeneidad" (BLANCO GUIJARRO, 2005, p. 10). Para esa autora, la educación común tiene la responsabilidad de atender a la diversidad, puesto que las diferencias son inherentes a los seres humanos, razón por la cual están presentes en todos los procesos de educación cuyo foco sea la enseñanza-aprendizaje.

\section{CONCLUSIONES}

Tras el término de esta investigación, aportaremos las conclusiones a que llegamos.

Para interpretar los datos relacionando los resultados en relación a los objetivos de esa investigación, recordamos que el objetivo general es: analizar el proceso de inclusión del niño con necesidades educativas especiales derivadas del déficit 
cognitivo en los salones de clase regulares de la Educación Infantil y de la Enseñanza Primaria en el municipio de Belém, en el Estado de Pará, en la Región Amazónica.

Los objetivos específicos son: verificar cómo la diversidad ha sido contemplada en las propuestas curriculares en cuestión; identificar el nivel de formación profesional de los docentes que trabajan con alumnos con necesidades educativas especiales e investigar las condiciones de organización y de infraestructura de las escuelas locales para acoger a esos niños.

En relación al objetivo general, concluimos que, en Belém, como en todo el Brasil, el discurso acerca de la inclusión de niños especiales en la escuela se ha propagado rápidamente entre todos los miembros de la comunidad escolar, pero la educación de esos niños aún es objeto de inquietudes, prejuicios y discriminaciones.

Muchos miembros de la comunidad escolar todavía ven el sujeto con discapacidad como un "alumno especial", cuyas necesidades específicas demandan equipos propios, muchos recursos y grandes niveles de especialización por parte de los profesores. O sea, tienen una visión con prejuicios del niño especial y muchas escuelas la realizan solamente para cumplir la ley.

Por causa de esos factores, la inclusión escolar aparece como un ideal utópico e inviable, pues a pesar de que la mayoría de los sujetos investigados creen que ella es viable, todos apuntan que la falta de capacitación de los profesores, de soporte técnico y apoyo especializado por parte de las escuelas y de un currículo flexible constituyen grandes trabas para la inclusión, haciéndola inviable.

Muchos sujetos la apuntan como un avance, aunque todavía esté sólo empezando, faltando aún mucho que aprender y desarrollar.

Sobre ese punto creemos que el principal avance acerca de la inclusión es el inicio de un proceso de concientización de la sociedad, pues antes no veíamos niños especiales en los salones de clase regulares y hoy ya vemos a algunos.

Otro objetivo a ser analizado consiste en verificar cómo la diversidad ha sido contemplada en las propuestas curriculares en cuestión.

Observamos que, de modo general en las escuelas investigadas, el currículo es el mismo para una clase inclusiva, sin llevar en consideración que el proceso 
cognitivo es más lento para una persona con necesidades especiales derivadas de déficit cognitivos, como es el caso tratado en esta pesquisa.

No observamos cualquier material pedagógico adaptado para atender las necesidades educacionales del niño especial y, a pesar de que muchos tienen el P.E.I (Plan de Enseñanza Individualizado), no vislumbramos cualquier preocupación del docente en seguir ese plan con el niño especial.

Además de eso, cuando preguntamos qué podría ser hecho para mejorar el proceso de inclusión, pocos profesores respondieron que se debería elaborar un currículo flexible, capaz de atender a todos.

El otro objetivo analizado en relación a los resultados es identificar el nivel de formación profesional de los docentes que trabajan con alumnos con necesidades educativas especiales.

Notamos que la mayoría de los profesores posee poca o ninguna experiencia en el área de la inclusión, pues como ya fue dicho, es un campo nuevo y, por eso, los propios docentes aún no se sienten preparados para cumplir las exigencias de la Ley de Directrices y Bases de la Educación Nacional (LDB) que exige la preparación de los pedagogos para dar atención a ese público.

Con eso se observa la falta de capacitación de esos docentes, que resaltan, entre otras dificultades, su falta de preparación para trabajar con la diversidad del grupo de alumnos y con la amplitud y la complejidad de los procesos de enseñanza y aprendizaje, además de la difícil realidad de las condiciones de trabajo, de la red física inadecuada, sin adaptaciones necesarias para trabajar con las diferencias. O sea, la mayoría se siente sin preparación para enseñar a "alumnos especiales" o diferentes, pues la formación de estos profesores se caracteriza por la cualificación específica, obtenidas por medio de cursos de pedagogía o de otros cursos de formación hechos en instituciones especializadas en educación "tradicional" que no enseñan a trabajar con la discapacidad, lo que limita sus posibilidades de actuación.

En relación a eso, algunos docentes investigados relatan que no saben cómo prepararse para esta actual perspectiva del sector, pues la principal dificultad está en el hecho de que el currículo universitario del profesional de educación no contempla asignaturas específicas del área de la inclusión de forma profundizada, lo que lleva a 
esos profesionales a buscar formación en cursos de postgrado que son impartidos la mayoría de veces en universidades privadas.

Así, formar educadores comprometidos, capacitados y actualizados con las nuevas exigencias de la LDB (Ley de Directrices y Bases) para los parámetros de la educación inclusiva es uno de los mayores desafíos actuales del sector pedagógico brasileño, de un modo general, y paraense, de forma específica pues, de acuerdo con la ley, los alumnos llamados "especiales" ahora tienen que estudiar en espacios integrados con otros colegas.

Finalmente el último objetivo analizado consiste en investigar las condiciones de organización y de infraestructura de las escuelas locales para acoger a esos niños.

Observamos que existe un déficit estructural en las escuelas locales para la adecuación del niño especial, pues no hay aplicación de muchos recursos en beneficio de la inclusión educacional, o sea, los problemas de infraestructura escolar son consecuencia de la falta de inversión en educación y en políticas públicas en la ciudad de Belém.

Por eso, no encontramos en ninguna de las escuelas investigadas infraestructura adecuada para recibir el alumno especial, pues ni todas tienen recursos didácticos y equipos multidisciplinares constituidos por psicólogos, fonoaudiólogos y psicopedagogos, indispensables para que la escuela se configure como un lugar de cambio en la perspectiva de la educación inclusiva, o sea que la escuela sufrió poca alteración al incluir alumnos especiales en su contexto.

Siendo así, la escuela deberá ser organizada para promover la innovación y los cambios en diferentes perspectivas y ofrecer respuestas educativas coherentes con las necesidades especiales de los alumnos.

Por consiguiente, es importante la participación de todos los miembros de la comunidad escolar en la organización de la escuela, por medio de proyectos educativos de carácter innovador.

Por todo lo que fue analizado, se concluye que existe una serie de contradicciones entre el discurso y la acción en relación a la experiencia de la inclusión, pues lo que se nota es que el discurso de los sujetos no coincide con la realidad observada, investigada y analizada. 
Pero ya constatamos un innegable cambio de actitudes de parte de los educadores y de la sociedad en general en relación a ese tema tan polémico.

Estas transformaciones se traducen en el reconocimiento y en la afirmación de los derechos de todos a la educación y en la asimilación de principios y metas que deben ser alcanzadas.

Tenemos que intentar ahora proponer acciones y medidas que tengan como objetivo concientizar a todos y asegurar los derechos conquistados, la mejoría de la calidad de la educación, la inversión en una amplia capacitación de los educadores para lidiar con las diferencias y la provisión de recursos materiales y humanos para equipar las escuelas para recibir a los "diferentes" y deben ser hechas adaptaciones curriculares para garantizar a todos los alumnos, incluso a los que presentan necesidades educacionales especiales, respuestas educativas que deben ser dadas por el sistema educacional.

En fin, se observa que, a pesar de los reconocidos avances ocurridos en relación a la educación inclusiva en Brasil y en Belém, aún son necesarias acciones más concretas por parte del gobierno y de toda la sociedad para que haya una efectiva actividad educativa regular en esa área.

A pesar de todo, no se puede negar las victorias ya conseguidas en dirección al reconocimiento y a la valoración de las diferencias. No obstante, para que los individuos especiales sean considerados ciudadanos, mucha cosa aún deben ser hechas, siendo necesaria una transición para que la inclusión pase a ser un sueño posible.

\section{REFERENCIAS}

BARROS, G. S. Políticas e práticas de inclusão na rede pública de ensino do município de João Pessoa - PB. Lisboa: Universidade Lusófona de Humanidades e Tecnologias. Departamento de Ciências Sociais e Humanas, 2005.

BLANCO GUIJARRO, M. R. Inclusão: um desafio para os sistemas educacionais. In: ENSAIOS pedagógicos: construindo escolas inclusivas. Brasília, DF: Secretaria de Educação Especial/MEC, 2005. p. 7-14. Disponible en: $<$ http://portal.mec.gov.br/seesp/arquivos/pdf/ensaiospedagogicos.pdf>. Acceso en: jul. 2010. 
CANEN, A.; XAVIER, G. P. M. Multiculturalismo, pesquisa e formação de professores: o caso das Diretrizes Curriculares para a formação docente. Ensaio: Avaliação e políticas públicas em educação. Rio de Janeiro: Fundação Cesgranrio, v. 13, n. 48, p. 333-334, jul./set. 2005. Disponible en: <http://revistas.cesgranrio.org.br/index.php/ensaio/rt/captureCite/723/0>. Acceso: jul. 2010.

FIGUEIREDO, R. V. A formação de professores para inclusão dos alunos no espaço pedagógico da diversidade. In: MANTOAN, M. T. E. (Org.). O desafio das diferenças nas escolas. Petrópolis: Vozes, 2008, v. 1, p. 141-145.

MANTOAN, M. T. E. A hora da virada. Inclusão - Revista da Educação Especial. Brasília, DF: Secretaria de Educação Especial/MEC, v. 1, n. 1, p. 24-28, out. 2005. . "Inclusão é o privilégio de conviver com as diferenças". Inclusive - inclusão e cidadania. 22 abr. 2008. Disponible en: <www.inclusive.org.br/arquivos/50>. Acceso en: jul. 2010.

REYES REBOLLO, M. M. La formación del profesorado: motor de cambio en la escuela del siglo XXI. Revista de Educación Inclusiva, v. 3, n. 3, p. 89-102, 2010. Disponible en: <http://www.ujaen.es/revista/rei/linked/documentos/documentos/117.pdf>. Acceso en: jul. 2010.

SÁNCHEZ, P. A. A educação inclusiva: um meio de construir escolas para todos no século XXI. Inclusão - Revista da Educação Especial. Brasília, DF: Secretaria de Educação Especial/MEC, v. 1, n. 1, p. 7-18, out. 2005. Disponible en: <http://portal.mec.gov.br/seesp/arquivos/pdf/revistainclusao1.pdf>. Acceso en: 20 sept. 2010 .

STAINBACK, S.; STAINBACK, W. (Orgs.). Inclusão: um guia para educadores. Porto Alegre: Artmed, 1999.

TORRES GONZÁLEZ, J. A. Educação e diversidade: bases didáticas e organizativas. Porto Alegre: Artmed, 2002.

Programa de intervención para alumnos con necesidades educativas de carácter cognitivo. Acción pedagógica, v. 10, n. 1-2, p. 74-84, 2001. 